\title{
ergopraxis unter den besten Drei!
}

_ Einmal jährlich zeichnet die Deutsche Fachpresse die besten deutschen Fachmedienangebote mit einem Preis aus. Eine Fachjury beurteilt dabei die eingereichten Zeitschriften nach insgesamt vier Kriterien: Gesamtkonzept, Inhalt, Gestaltung und Kommunikation.

\section{SHORTLIST 2010 \\ Kategorie: Medizin/Pharma/ Gesundheit \\ FACHMEDIUM DES JAHRES PREIS DER DEUTSCHEN FACHPRESSE}

Deutsche Fachpresse
_ Von Null auf Hundert schaffte es ergopraxis in der Kategorie „Medizin, Pharma, Gesundheit“ unter die besten Drei - und damit auf die sogenannte Shortlist. Das macht uns in der Redaktion natürlich mächtig stolz!
_ Auf diesen Lorbeeren werden wir uns allerdings nicht ausruhen. Im Gegenteil: Wir stellen unsere Zeitschrift kontinuierlich auf den Prüfstand, drehen an gestalterischen Schräubchen, polieren die ein oder andere Rubrik auf und gestalten unser Heft damit für Sie noch attraktiver.

_-Wenn Sie bei unserem Feintuning einen Werksfehler entdecken, finden Sie für Ihre Anregungen, Ihr Lob und natürlich auch für Ihre Kritik ein offenes Ohr. Schreiben Sie mir gerne eine E-Mail, ich freue mich über Ihr Feedback!

Herzlichst

Ihre

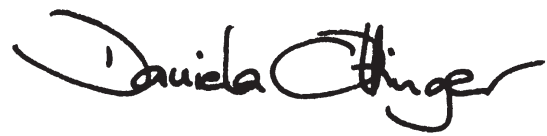

\section{ZU GEWINNEN}

Kursplatz

1 Orthopädisch-traumatologischer Finger- und Handkurs Seite 45

Bücher

5 Doppelpacks des Buchs „Sprich mit mir!“ und der CD „Sing mit mir! Sprich mit mir!“" Seite 18 2-mal „Borderline - die andere Art zu fühlen“" Seite 22

3 Doppelpacks der Fallbücher „Ergotherapie in der Psychiatrie“ und „Ergotherapie in der Pädiatrie"

Seite 31

Im ergopraxis.Refresher

2-mal „Training mit aufmerksamkeitsgestörten Kindern“ $\quad$ Seite 15 